\title{
Research Article \\ Performance Assessment of a Freight Network with Stochastic Capacities
}

\author{
Xiu-Zhen Xu $\mathbb{D}^{1},{ }^{1}$ Yi-Feng $\mathrm{Niu} \mathbb{D}^{2},{ }^{2}$ and Qing $\mathrm{Li}^{3}$ \\ ${ }^{1}$ School of Computer Science and Technology, Henan Polytechnic University, Jiaozuo 454000, China \\ ${ }^{2}$ School of Mathematics and Information Science, Henan Polytechnic University, Jiaozuo 454000, China \\ ${ }^{3}$ College of Mathematics and Systems Science, Shandong University of Science and Technology, Qingdao 266590, China
}

Correspondence should be addressed to Yi-Feng Niu; ifeng021@126.com

Received 27 January 2018; Revised 4 August 2018; Accepted 26 August 2018; Published 18 September 2018

Academic Editor: Daniela Paolotti

Copyright (c) 2018 Xiu-Zhen Xu et al. This is an open access article distributed under the Creative Commons Attribution License, which permits unrestricted use, distribution, and reproduction in any medium, provided the original work is properly cited.

\begin{abstract}
This paper focuses on performance assessment of a freight network with stochastic capacities by using reliability analysis, in which a node denotes a supplier, a transfer center, a distribution center, or a market, while an arc denotes a logistics service provider offering the freight traffic service for a pair of nodes. Due to some uncertainties in real environment (for example, a proportion of vehicles owned by the provider may be reserved by other customers), the available capacity of the provider along each arc is stochastic. Thus, network reliability that at least $d$ units of commodity demand can be successfully delivered from the source to the destination denotes the operational performance of such a freight network. A $d$-minimal path-based method is developed to evaluate the freight network reliability. To advance the efficiency of solving $d$-minimal paths, an improved model is established by redefining capacity bounds of arcs and minimal paths. Furthermore, a new concept of expected capacity grounded on network reliability is presented to measure the service performance of the freight network. A practical case related to the coal delivery network is studied to demonstrate the implications of both network reliability and expected capacity.
\end{abstract}

\section{Introduction}

From the perspective of logistics operation and management, it is vitally important to design a robust freight network that runs as well as possible under normal conditions, while also running relatively well under unexpected situations. Performance assessment is an effective manner to capture the operational state of freight networks in a complex environment and thus plays a critical role in logistics management. Traditionally, most studies [1-5] have studied the performance of freight/logistics networks from several aspects, such as meeting customer needs, maximizing customer service levels, minimizing total costs, or ensuring timely delivery, in which market demand, service level, lead time, or distribution cost are the concerned factors directly effecting the performance of freight networks. However, the studied networks in the aforementioned literature are essentially deterministic. In reality, a freight network is always affected by various unexpected events, such that its capacity is subject to degradation. Therefore, whether the freight network is able to ensure the delivery of required quantity of goods from the supply point to the demand point is of utmost importance to both suppliers and customers. To guarantee an effective logistics performance, the key is to know the level of operation of the freight network both in stable and uncertain states [6].

A freight network is composed of several nodes and arcs, where each node stands for a supplier, a transfer center, a distribution center, or a market, and each arc (route) linking a pair of nodes stands for a logistics service provider offering the freight traffic service. Since the capacity (the number of cars or trucks) of a provider may be reserved by other customers, its available capacity in the freight network is stochastic in nature. For example, it is assumed that the maximal capacity of a logistics service provider is three trucks. If all trucks are reserved by other customers, the available capacity is 0 ; if one truck is reserved, the available capacity is 2 ; if two trucks are reserved, the available capacity is 1 . In this sense, the actual capacity of each provider in the freight network is looked upon as a random variable, following a specified probability distribution. Therefore, a freight 
network can be modeled as a typical stochastic-flow network, in which each arc has stochastic capacities, and the delivered commodities are considered as a flow [7-13].

Reliability evaluation is a powerful tool for the demonstration of network performance. For a stochastic-flow network, the reliability index $R_{d}$ is defined as the probability that at least $d$ units of flow (i.e., commodity demand) can be successfully transmitted from the source to the destination through stochastic arc capacities [14-22]. To date, many studies $[14-18,21,22]$ have evaluated the reliability $R_{d}$ in terms of $d$-minimal paths. A $d$-minimal path $x$ is a minimal capacity vector meeting the demand level $d$, which means that for any $y<x, y$ does not meet the demand level $d$ [14]. When all $d$-minimal paths are at hand, the inclusionexclusion rule is available to calculate $R_{d}[14,15]$.

There are two types of models reported to solve $d$-minimal paths. One is first proposed by Lin et al. [14], and the other is originally proposed by Yeh [17]. Note that the model of Yeh [17] is built on the well-known max-flow mathematical programming model and is more applicable to directed networks (if it is used to solve undirected networks, an additional step of transforming undirected networks into directed networks is required [21]). Provided that all minimal paths are known, the model of Lin et al. [14] is based on the network structure and the flow-conservation law. A path is a sequence of arcs that connects the source node to the destination node, and a minimal path is such a path that removing any arc will make it no longer a path. The model by Lin et al. applicable to solving both directed networks and undirected networks is widely used by the existing algorithms $[14-16,22]$ to search for $d$-minimal paths. For example, Lin [15] proposed a method to solve $d$-minimal paths of a network with unreliable nodes; Yeh [16] proposed a cycle-checking method to verify whether a feasible solution to the model by Lin et al. is a $d$-MP; Chen and Lin [22] considered to utilize the fast enumeration method to solve $d$-minimal paths. To be worthy of attention, some constraints in the model of Lin et al. [14] are so relaxed that a large number of feasible solutions need to be checked in order to find all $d$-minimal paths, which to a large extent effects the computational efficiency.

This paper concentrates on performance assessment of a freight network with stochastic capacity by using reliability analysis. Specifically, the major contributions of this paper are twofold. First, by redefining capacity bounds of arcs and minimal paths, we construct an improved model based on which an efficient algorithm is suggested to solve $d$-minimal paths. Both complexity analysis and illustrative examples are provided to show the benefit of the proposed algorithm. As demonstrated through examples, the proposed algorithm compares favorably with the existing methods. Second, we propose a new concept of expected capacity grounded on network reliability to assess the service performance of a freight network. Thus, the performance level of a freight network with stochastic capacities can be evaluated in terms of either network reliability or expected capacity. A practical case study related to a coal delivery network is provided to illustrate the utility of the proposed algorithm and demonstrate the implications of both network reliability and expected capacity.
The remainder of this paper is organized as follows. Section 2 introduces the stochastic-flow network model and some basics for reliability evaluation. In Section 3, the fundamental model is first discussed, and then an improved model is constructed by redefining capacity bounds of arcs and minimal paths. An algorithm for solving $d$-minimal paths is presented in Section 4, along with discussions on its time complexity. A simple illustrative example and a numerical example are also provided in Section 4. A concept of expected capacity is put forward in Section 5. In Section 6, a practical case study is provided to explain the implications of both network reliability and expected capacity. Section 7 presents some concluding remarks.

\section{Preliminaries}

2.1. Stochastic-Flow Network. Theoretically, a freight network with stochastic capacities can be regarded as a typical stochastic-flow network. Let $G(V, E, W)$ represent a stochastic-flow network, where $V=\{s, 1,2, \ldots, n, t\}$ is the set of nodes with $s$ (source node) denoting the supply point, $t$ (destination node) denoting the demand point, and $n$ denoting the number of nodes except $s$ and $t, E=$ $\left\{e_{1}, e_{2}, \ldots, e_{m}\right\}$ is the set of arcs (routes) with $m$ denoting the number of arcs, where $e_{i}(1 \leq i \leq m)$ is the $i$ th arc connecting a pair of nodes, and $W=\left(W_{1}, W_{2}, \ldots, W_{m}\right)$ is the largest capacity vector with $W_{i}(1 \leq i \leq m)$ being the largest capacity of arc $e_{i}$. Along each arc, a contracted logistics service provider is responsible for freight traffic. The available capacity of each logistics service provider is a random variable notated by $x_{i}$, and $x_{i}$ takes integer values from 0 to $W_{i}$ according to a given probability distribution. Note that the data with respect to capacity and its probability distribution can be obtained from the database of logistics service providers.

A capacity vector $x=\left(x_{1}, x_{2}, \ldots, x_{m}\right)$ indicates the current capacity of each arc, and $M(x)$ denotes the max-flow of the network under $x$, i.e., the capacity of the freight network under $x$. As with the existing literature [7-13], the following assumptions are considered:

(1) All flows in the network satisfy the flow-conservation law, i.e., total flows into and from a node (other than the source and destination nodes) are all equal

(2) The capacities of different arcs (logistics service providers) are statistically independent

(3) Flow in the network is an integer value

(4) Each node is perfectly reliable, which means no constraint is imposed on the capacities of nodes (transfer center or distribution center is responsible for the transshipment service only)

For example, Figure 1 that is cited from [21] presents a simple freight network with $V=\{s, 1,2, t\}, n=2, E=\left\{e_{1}, e_{2}\right.$, $\left.e_{3}, e_{4}, e_{5}\right\}$, and $m=5$. The capacity and capacity probability distribution of each arc are given in Table 1 indicating $W=(3,2,1,2,2)$. Given a capacity vector $x=(2,1,0,1,2)$ 


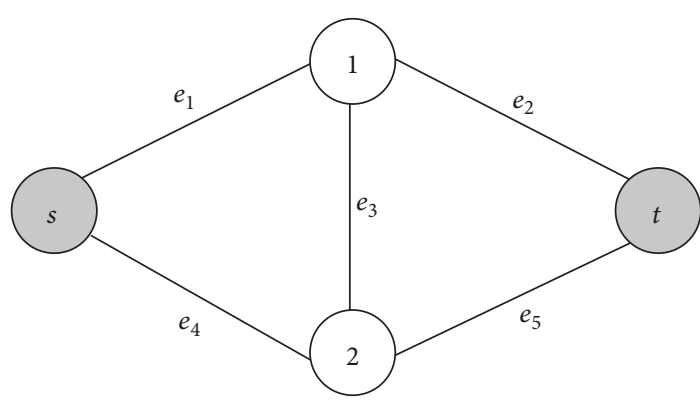

Figure 1: A simple freight network.

TABLE 1: The data of arcs in Figure 1.

\begin{tabular}{llllllllc}
\hline Arc & \multicolumn{3}{c}{ Capacities } & \multicolumn{5}{c}{ Capacity probabilities } \\
\hline$e_{1}$ & 0 & 1 & 2 & 3 & 0.05 & 0.10 & 0.25 & 0.60 \\
$e_{2}$ & 0 & 1 & 2 & - & 0.10 & 0.30 & 0.60 & - \\
$e_{3}$ & 0 & 1 & - & - & 0.10 & 0.90 & - & - \\
$e_{4}$ & 0 & 1 & 2 & - & 0.05 & 0.25 & 0.70 & - \\
$e_{5}$ & 0 & 1 & 2 & - & 0.10 & 0.20 & 0.70 & - \\
\hline
\end{tabular}

which indicates the current capacities of $e_{1}, e_{2}, e_{3}, e_{4}$, and $e_{5}$ are $2,1,0,1$, and 2 , respectively, the max-flow of the network under $x$ is $M(x)=2$.

2.2. Reliability Evaluation in terms of d-Minimal Paths. As stated previously, the reliability $R_{d}$ that serves as a performance index of a freight network is defined as the probability that at least $d$ units of commodity demand can be successfully delivered from the source to the destination, then $R_{d}=\operatorname{Pr}$ $\{x \mid M(x) \geq d\}$. Obviously, it is extremely time-consuming to calculate $R_{d}$ by directly checking $M(x) \geq d$ for every capacity vector $x$ due to the large number of capacity vectors. Alternatively, most studies have focused on the $d$-minimal path-based method. A capacity vector $x=\left(x_{1}, x_{2}, \ldots, x_{m}\right)$ is a $d$-minimal path if and only if (1) $M(x)=d$ and (2) $M\left(x-0\left(e_{i}\right)\right)<d$ for each $x_{i}>0$, where $0\left(e_{i}\right)=(0, \ldots, 0,1$, $0, \ldots, 0)$, i.e., the capacity level is 1 for $e_{i}$ and 0 for other arcs [14]. The definition implicitly demonstrates that a $d$-minimal path is the minimal capacity vector satisfying the demand level $d$. If all $d$-minimal paths are found, $R_{d}$ can be calculated by the well-known inclusion-exclusion method.

Assuming $y^{1}, y^{2}, \ldots, y^{\sigma}$ are all $d$-minimal paths and let $B_{1}=\left\{x \mid x \geq y^{1}\right\}, B_{2}=\left\{x \mid x \geq y^{2}\right\}, \ldots, B_{\sigma}=\left\{x \mid x \geq y^{\sigma}\right\}$, where $x=\left(x_{1}, x_{2}, \ldots, x_{m}\right), y^{i}=\left(y_{1}^{i}, y_{2}^{i}, \ldots, y_{m}^{i}\right)$, and $x \geq y^{i}$ means that $x_{j} \geq y_{j}^{i}$ for $j=1,2, \ldots, m$, then $R_{d}$ can be evaluated via the inclusion-exclusion method as follows:

$$
\begin{aligned}
R_{d}= & \operatorname{Pr}\{x \mid M(x) \geq d\} \\
= & \operatorname{Pr}\left(B_{1} \bigcup B_{2} \bigcup \ldots \bigcup B_{\sigma}\right) \\
= & \sum_{i=1}^{\sigma} \operatorname{Pr}\left(B_{i}\right)-\sum_{j=2}^{\sigma} \sum_{i=1}^{j-1} \operatorname{Pr}\left(B_{i} \bigcap B_{j}\right)+\cdots \\
& +(-1)^{\sigma-1} \operatorname{Pr}\left(B_{1} \bigcap B_{2} \bigcap \ldots \bigcap B_{\sigma}\right),
\end{aligned}
$$

where $\operatorname{Pr}\left\{B_{i}\right\}=\operatorname{Pr}\left\{x \mid x \geq y^{i}\right\}=\prod_{k=1}^{m} \operatorname{Pr}\left\{x_{k} \geq y_{k}^{i}\right\}, \operatorname{Pr}\left\{B_{i} \cap\right.$ $\left.B_{j}\right\}=\operatorname{Pr}\left\{x \mid x \geq \max \left\{y^{i}, y^{j}\right\}\right\}=\prod_{k=1}^{m} \operatorname{Pr}\left\{x_{k} \geq \max \left\{y_{k}^{i}, y_{k}^{j}\right\}\right\}$, $\ldots, \operatorname{Pr}\left(B_{1} \cap B_{2} \cap \cdots \cap B_{\sigma}\right)=\operatorname{Pr}\left\{x \mid x \geq \max \left\{y^{1}, y^{2}, \ldots, y^{\sigma}\right\}\right\}$ $=\prod_{k=1}^{m} \operatorname{Pr}\left\{x_{k} \geq \max \left\{y_{k}^{1}, y_{k}^{2}, \ldots, y_{k}^{\sigma}\right\}\right\}$.

Therefore, the $d$-minimal path problem plays the key role in the network reliability evaluation and will be the focus in the subsequent discussions.

\section{An Improved Model with respect to $d$ -Minimal Paths}

3.1. The Basic Model. As mentioned before, the model proposed by Lin et al. [14] is one of the two fundamental models adopted to solve $d$-minimal paths. In this section, we will make some efforts to improve the model by Lin et al. It is assumed that there are $p$ minimal paths, say, $P_{1}, P_{2}, \ldots, P_{p}$, from the source node to the destination node in the network. The flow through $P_{j}(1 \leq j \leq p)$ is denoted by $F_{j}(1 \leq j \leq p)$. $F=\left(F_{1}, F_{2}, \ldots, F_{p}\right)$ that consists of flows through all minimal paths is called a flow vector. The model of Lin et al. is built on the following lemma [14-16, 22].

Lemma 1. Given the demand level $d$, a capacity vector $x=$ $\left(x_{1}, x_{2}, \ldots, x_{m}\right)$ is called a d-minimal path candidate if it satisfies the following conditions:

$$
\begin{gathered}
F_{1}+F_{2}+\cdots+F_{p}=d, \\
0 \leq F_{j} \leq \mathrm{CP}_{j} \quad \text { for } 1 \leq j \leq p, \\
0 \leq \sum_{e_{i} \in P_{j}} F_{j} \leq W_{i} \quad \text { for } 1 \leq i \leq m, \\
x_{i}=\sum_{e_{i} \in P_{j}} F_{j} \quad \text { for } 1 \leq i \leq m,
\end{gathered}
$$

where $\mathrm{CP}_{j}=\min \left\{W_{i} \mid e_{i} \in P_{j}\right\}$ is the capacity of minimal path $P_{j}$. Equation (2) shows that the summation of flows through all minimal paths must be equal to demand level $d$, condition (3) points out that the flow through $P_{j}$ should not exceed the capacity of $P_{j}$, and condition (4) indicates that the flow through $e_{i}$ should not be above the largest capacity of $e_{i}$. Equation (5) specifies the relationship between the current capacity of $e_{i}$ and the flow through $e_{i}$. It should be noted that the feasible solution derived from Lemma 1 is a d-minimal path candidate [14]. Each d-minimal path is a $d$-minimal path candidate, but a d-minimal path candidate is not necessarily a d-minimal path. Therefore, a d-minimal path candidate needs to be checked to further determine whether it is a d-minimal path or not. The popular methods for checking d-minimal path candidates include the comparison method [14, 15] and the cycle-checking method [16]. The comparison method is easy to understand and is based on the following lemma $[14,15]$.

Lemma 2. For a $d$-minimal path candidate $x$, if there exists no d-minimal path candidate $y$ such that $x>y$, where $x>y$ 
means $x_{i} \geq y_{i}$ for $i=1,2, \ldots, m$ and $x_{j}>y_{j}$ for at least one $j(1 \leq j \leq m)$, then $x$ is a d-minimal path.

3.2. The Improved Model. A flow vector $F=\left(F_{1}, F_{2}, \ldots, F_{p}\right)$ satisfying conditions (2)-(4) is said to be a feasible flow vector. Lemma 1 shows that it first needs to find all of the feasible flow vectors, and then each feasible flow vector is transformed into its corresponding $d$-minimal path candidate by (5). Note that when the cost of solving feasible flow vectors is decreased, the efficiency of solving $d$-minimal paths would be improved. As can be seen below, the lower bounds in condition (4) have the potential to be raised, and the upper bounds in conditions (3) and (4) have the potential to be dropped.

Let $W\left(0_{i}\right)$ denote a special capacity vector in which the capacity level is 0 for $e_{i}$, and the capacity level is the largest capacity for other arcs, i.e., $W\left(0_{i}\right)=\left(W_{1}, W_{2}, \ldots, W_{i-1}, 0\right.$, $\left.W_{i+1}, \ldots, W_{m}\right)$ and $L_{i}=\max \left\{0, d-M\left(W\left(0_{i}\right)\right)\right\}$, then the following theorem clearly presents the necessary condition for a capacity vector to be a $d$-minimal path.

Theorem 1. Given the demand level $d$, if a capacity vector $x=\left(x_{1}, x_{2}, \ldots, x_{m}\right)$ is a d-minimal path, then $x_{i} \geq L_{i}$ for $1 \leq i \leq m$.

Proof 1. Clearly, $L_{i} \geq 0$ holds. If $L_{i}=0$, it is easy to have $x_{i} \geq L_{i}$.

If $L_{i}>0$, it means $L_{i}=d-M\left(W\left(0_{i}\right)\right)>0$. First, it is noted that $M(W) \geq d$. Thus, when $d-M\left(W\left(0_{i}\right)\right)>0$, i.e., $M\left(W\left(0_{i}\right)\right)<d$, it means that at least $d-M\left(W\left(0_{i}\right)\right)$ units of flow must travel through arc $e_{i}$ in order for $d$ units of flow to be transmitted from the source node to the destination node. If $x$ is a $d$-minimal path, $M(x)=d$ holds, i.e., $d$ units of flow can be transmitted from the source node to the destination node under $x$. As a result, it is deduced that $x_{i} \geq d-M\left(W\left(0_{i}\right)\right)=L_{i}$.

In Theorem $1, L_{i}$ can be looked upon as a new lower capacity bound of $e_{i}$, and thus it is used in condition (4) to reduce the cost of solving feasible flow vectors. Because computing $M\left(W\left(0_{i}\right)\right)$ requires $O\left(n^{3}\right)$ time $[23,24]$, the time complexity of determining $L_{i}$ is also $O\left(n^{3}\right)$. Therefore, the total time complexity of finding all lower bounds in condition (4) is $O\left(m n^{3}\right)$.

Corollary 1. The time complexity of finding all lower bounds in condition (4) is $O\left(\mathrm{mn}^{3}\right)$.

It should be pointed out that the time complexity $O\left(m n^{3}\right)$ of finding all lower bounds, i.e., Corollary 1 , is far lower than the one for solving $d$-minimal path candidates, so it has no influence on the whole algorithm. Additionally, notice that $F_{1}+F_{2}+\cdots+F_{p}=d$ in condition (2) means $F_{j} \leq d$ for $1 \leq j \leq p$, and it is easy to know $\sum_{e_{i} \in P_{j}} F_{j} \leq F_{1}+F_{2}+\cdots+$ $F_{p}=d$; hence, the upper bounds in conditions (3) and (4) can be replaced by $\min \left\{\mathrm{CP}_{j}, d\right\}$ and $\min \left\{W_{i}, d\right\}$, respectively. Grounding on Theorem 1 and the above analyses, the following improved model can be constructed to solve all $d$-minimal path candidates.
Theorem 2. Given the demand level $d$, a capacity vector $x=$ $\left(x_{1}, x_{2}, \ldots, x_{m}\right)$ is called a d-minimal path candidate if it satisfies the following conditions:

$$
\begin{gathered}
F_{1}+F_{2}+\cdots+F_{p}=d, \\
0 \leq F_{j} \leq \min \left\{\mathrm{CP}_{j}, d\right\} \quad \text { for } 1 \leq j \leq p, \\
L_{i} \leq \sum_{e_{i} \in P_{j}} F_{j} \leq \min \left\{W_{i}, d\right\} \quad \text { for } 1 \leq i \leq m, \\
x_{i}=\sum_{e_{i} \in P_{j}} F_{j} \text { for } 1 \leq i \leq m .
\end{gathered}
$$

Proof 2. Directly from Theorem 1 and Lemma 1.

Obviously, Theorem 2 is an improvement to Lemma 1 in view of the tighter capacity constraints imposed on $e_{i}$ (corresponding to conditions (7) and (8)), and it is the foundation of the designed algorithm for solving $d$-minimal paths in this paper.

\section{An Algorithm for Solving $d$-Minimal Paths}

4.1. The Developed Algorithm. Like the algorithms in [14-16, 22], it is assumed that all minimal paths are known in advance. Actually, there have already been many efficient methods to find minimal paths, such as Chen and Lin [25], Bai et al. [26], and Yeh [27]. The proposed algorithm for solving $d$-minimal paths consists of three steps as in Algorithm 1.

Step 0 is a preprocessing step for computing lower and upper bounds in conditions (7) and (8). Step 1 is the most important step for solving $d$-minimal path candidates. As the fast enumeration method proposed by Chen [28] has proven to be more efficient than the traditional enumeration method, it is suggested to solve all of the feasible flow vectors in Step 1. The detailed procedure of the fast enumeration method can be found in [22]. Step 2 is to check whether the derived $d$-minimal path candidates are indeed $d$-minimal paths. The time complexity of every step is discussed as follows. In Step 0, it takes $O(\mathrm{mp})$ time and $O(m)$ time to compute all $\min \left\{\mathrm{CP}_{j}, d\right\}(1 \leq j \leq p)$ and all min $\left\{W_{i}, d\right\}(1 \leq i \leq m)$, respectively. Computing all $L_{i}=\max$ $\left\{0, d-M\left(W\left(0_{i}\right)\right)\right\}(1 \leq i \leq m)$ requires $O\left(m n^{3}\right)$ time. For a general connected network, $O(p)=O\left(2^{n}\right)$ [29] means $O\left(m n^{3}\right)<O(m p)$; thus, Step 0 totally requires $O(m p)$ time. According to Chen and Lin [22], it takes $O\left(\prod_{i=1}^{\pi} q_{k}\right)$ time to generate all feasible flow vectors where $\pi$ is the number of groups of alternative orders arranged by the fast enumeration method and $q_{k}$ is the total number of enumerations in the $i$ th group. It takes $O(m \lambda p)$ to transform all feasible flow vectors into $d$-minimal path candidates where $\lambda$ is the number of $d$-minimal paths. As a result, the time complexity of Step 1 for solving $d$-minimal path candidates is $O\left(\prod_{i=1}^{\pi} q_{k}\right)+O(m \lambda p)$. Step 2 requires $O\left(m \lambda^{2}\right)$ time to check $d$-minimal path candidates in the worst case.

To demonstrate the advantage of the proposed algorithm, there is a need to compare with the existing methods. Given that the method recently reported by Chen and Lin [22] is 
Input: A stochastic-flow network $G(V, E, W)$ with demand level $d$.

Output: All $d$-minimal paths.

Step 0. Compute $\min \left\{\mathrm{CP}_{j}, d\right\}$ for $1 \leq j \leq p, \min \left\{W_{i}, d\right\}$ and $L_{i}=\max \left\{0, d-M\left(W\left(0_{i}\right)\right)\right\}$ for $1 \leq i \leq m$.

Step 1. Use the enumeration algorithm to solve all of the feasible flow vectors satisfying conditions (6)-(8), and transform each feasible flow vector into its corresponding $d$-minimal path candidate by Equation (9). Suppose $x^{1}, x^{2}, \ldots, x^{\lambda}$ are all the obtained $d$-minimal path candidates.

Step 2. Use the comparison method to check whether $x^{i}(1 \leq i \leq \lambda)$ is a $d$-minimal path or not.

(2.1) $\mathrm{I}=\Phi$ (stores the index of each non $d$-minimal path after checking).

(2.2) For $i=1$ to $\lambda$ and $i \notin \mathrm{I}$.

(2.3) For $j=2$ to $\lambda$ and $j \notin \mathrm{I}$.

(2.4) If $x^{j}<x^{i}, x^{i}$ is not a $d$-minimal path, $\mathrm{I}=\mathrm{I} \bigcup i$ and go to (2.7); if $x^{j} \geq x^{i}, x^{j}$ is not a $d$-minimal path, $\mathrm{I}=\mathrm{I} \bigcup j$ and go to (2.7).

(2.5) $j=j+1$.

(2.6) $x^{i}$ is a $d$-minimal path.

(2.7) $i=i+1$.

Algorithm 1

considered to be efficient in solving $d$-minimal paths, we compare the proposed algorithm with it. Of particular note is that the step of solving $d$-minimal path candidates is the primary difference between the proposed algorithm and the method by Chen and Lin, i.e., the proposed algorithm and the method by Chen and Lin utilize Theorem 2 and Lemma 1 , respectively, to solve $d$-minimal path candidates. Also, the burden of solving $d$-minimal path candidates is the bottleneck of the $d$-minimal path algorithm [22]. Therefore, we compare both algorithms in terms of the time complexity of solving $d$-minimal path candidates. According to the solution procedure of the fast enumeration method $[22,28]$, the number of enumerations $q_{k}$ implemented by the proposed algorithm is dependent on the capacity ranges in conditions (7) and (8), whereas the number of enumerations $q_{k}^{*}$ implemented by Chen and Lin's method is dependent on the capacity ranges in conditions (3) and (4). Because the capacity ranges in conditions (7) and (8) are smaller or equal to the ones in conditions (3) and (4), we have $q_{k} \leq q_{k}^{*}$, which means $O\left(\prod_{i=1}^{\pi} q_{k}\right) \leq O\left(\prod_{i=1}^{\pi} q_{k}^{*}\right)$ holds. Consequently, the time complexity of the proposed algorithm is upper bounded by that of Chen and Lin's method [22] in solving $d$-minimal path candidates.

4.2. An Illustrative Example. The network in Figure 1 is adopted to demonstrate how the proposed algorithm works to compute the network reliability. Figure 1 has four minimal paths from $s$ to $t: P_{1}=\left\{e_{1}, e_{2}\right\}, P_{2}=\left\{e_{1}, e_{3}\right.$, $\left.e_{5}\right\}, P_{3}=\left\{e_{4}, e_{5}\right\}, P_{4}=\left\{e_{4}, e_{3}, e_{2}\right\}$. Given the demand level $d=3$, the reliability $R_{3}$, i.e., the probability of 3 units of commodity demand being successfully delivered from $s$ to $t$, can be calculated using all 3-minimal paths that are obtained as follows.

Step 0. $\min \left\{\mathrm{CP}_{1}, d\right\}=\min \{2,3\}=2$, similarly, $\min \left\{\mathrm{CP}_{2}\right.$, $d\}=1, \min \left\{\mathrm{CP}_{3}, d\right\}=2, \min \left\{\mathrm{CP}_{4}, d\right\}=1 ; \min \left\{W_{1}, d\right\}=$ $\min \{3,3\}=3$, similarly, $\min \left\{W_{2}, d\right\}=2, \min \left\{W_{3}, d\right\}=1$, $\min \left\{W_{4}, d\right\}=2, \min \left\{W_{5}, d\right\}=2 ; L_{1}=\max \{0, d-M(W$ $\left.\left.\left(0_{i}\right)\right)\right\}=\max \{0,1\}=1$, similarly, $L_{2}=1, L_{3}=0, L_{4}=0, L_{5}=1$.
Step 1. Use the fast enumeration method to solve all of the feasible flow vectors satisfying the following conditions:

$$
\begin{aligned}
F_{1} & +F_{2}+F_{3}+F_{4}=3, \\
& 0 \leq F_{1} \leq 2, \\
& \leq F_{2} \leq 1, \\
& 0 \leq F_{3} \leq 2, \\
& \leq F_{4} \leq 1, \\
& \leq F_{1}+F_{2} \leq 3, \\
1 & \leq F_{1}+F_{4} \leq 2, \\
0 & \leq F_{2}+F_{4} \leq 1, \\
0 & \leq F_{3}+F_{4} \leq 2, \\
1 & \leq F_{2}+F_{3} \leq 2 .
\end{aligned}
$$

And the solution results are $F^{1}=(1,0,1,1), F^{2}=(1,0$, $2,0), F^{3}=(1,1,1,0), F^{4}=(2,0,1,0)$, and $F^{5}=(2,1,0,0)$. Therefore, by (9), the corresponding $d$-minimal path candidates are $x^{1}=(1,2,1,2,1), x^{2}=(1,1,0,2,2), x^{3}=(2,1,1$, $1,2), x^{4}=(2,2,0,1,1)$, and $x^{5}=(3,2,1,0,1)$.

Step 2. Use the comparison method to check whether $x^{i}$ $(1 \leq i \leq 4)$ is a $d$-minimal path or not.

(2.1) $I=\Phi$.

(2.2) $i=1$ and $i \notin I$.

(2.3) $j=2$ and $j \notin I$.

(2.4) $x^{2}=(1,1,0,2,2)<x^{1}=(1,2,1,2,1)$ is false, $x^{2} \geq x^{1}$ is false.

(2.5) $j=3$ and $j \notin I$.

Finally, there are five 3-minimal paths obtained as follows: $x^{1}=(1,2,1,2,1), x^{2}=(1,1,0,2,2), x^{3}=(2,1,1,1,2)$, $x^{4}=(2,2,0,1,1)$, and $x^{5}=(3,2,1,0,1)$. Let $B_{1}=\{x \mid x \geq$ $\left.x^{1}\right\}, \quad B_{2}=\left\{x \mid x \geq x^{2}\right\}, \quad B_{3}=\left\{x \mid x \geq x^{3}\right\}, \quad B_{4}=\left\{x \mid x \geq x^{4}\right\}$, and $B_{5}=\left\{x \mid x \geq x^{5}\right\}$, then it is trivial to derive $R_{3}=$ 0.6674025 by Equation (1). 


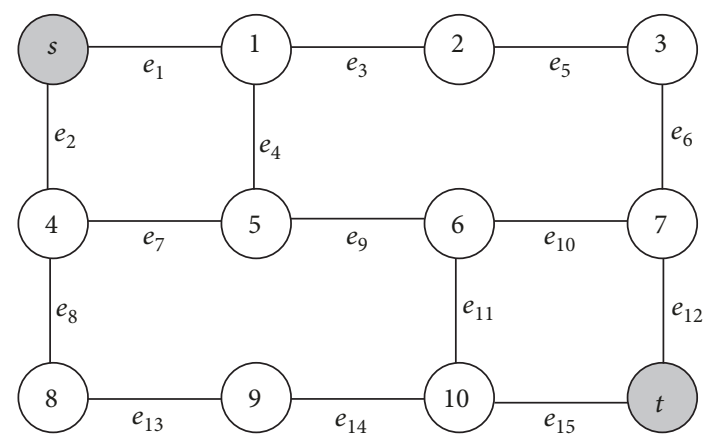

Figure 2: A benchmark network for the numerical example.

Furthermore, it is calculated that the proposed algorithm needs to perform 21 enumerations during the solution of 3minimal path candidates, i.e., solving conditions (10)-(19). By contrast, if the method by Chen and Lin [22] is used to solve 3-minimal path candidates of the network, it needs to perform a total of 28 enumerations. Hence, the suggested algorithm implements a smaller number of enumerations in comparison with Chen and Lin's method, as already illustrated by theoretical analyses in Section 3.1.

4.3. A Numerical Example. In this section, the efficiency of the proposed algorithm is further explored through a numerical example. As stated previously, the major difference between the proposed algorithm and the method by Chen and Lin [22] is the step for solving $d$-minimal path candidates; therefore, it is only necessary to compare both algorithms in terms of the efficiency of solving $d$-minimal path candidates. Both algorithms coded in a MATLAB program run on a PC with Intel (R) Core (TM) i5$3210 \mathrm{M} 2.50 \mathrm{GHz}$ CPU.

A medium-sized network shown in Figure 2 is adopted to conduct numerical experiments. The capacities of all arcs in Figure 2 are set to 8, then the largest network capacity is $D=16$. All of the $d$-minimal path candidates corresponding to eight demand levels ranging from $d=9$ to $d=16$ are solved. We focus on the required computational time for solving $d$-minimal path candidates for each demand level. The overall results are summarized in Table 2. From Table 2, it can be observed that the suggested algorithm displays an advantage over the method by Chen and Lin [22]. Meanwhile, note that the efficiency difference of both algorithms is negligible when demand level $d$ ranges from $d=9$ to $d=12$, but it becomes prominent when the demand level $d$ is above 12 .

\section{Expected Capacity}

It is evident that network reliability is a probabilistic performance index for characterizing the capability of a freight network to ensure the accurate delivery of required quantity of commodity from the source to the destination. In this section, a new performance indicator will be presented to evaluate a freight network. Because the network capacity of a freight network is a random variable whose value ranges from the smallest capacity 0 to the largest capacity $M(W)$, i.e., $0,1, \ldots, M(W)$, we define a concept of expected capacity from the perspective of mathematical expectation to measure the service performance of the freight network. Expected capacity notated as EC represents the average network capacity level and is defined as follows:

$$
\mathrm{EC}=\sum_{d=1}^{D} \operatorname{Pr}\{x \mid M(x)=d\} \times d
$$

where $D=M(W)$ is the largest network capacity and $\operatorname{Pr}$ $\{x \mid M(x)=d\}$ is the probability that network capacity is equal to $d$. Note that $d=0$ does not contribute to expected capacity, so the value of $d$ ranges from 1 to $D$.

Obviously, computing $\operatorname{Pr}\{x \mid M(x)=d\}$ is the key to obtaining EC. Given the demand level $d$, reliability index $R_{d}$ is the probability that network capacity is greater than or equal to $d$, i.e., $R_{d}=\operatorname{Pr}\{x \mid M(x) \geq d\}$; hence, a clear relationship between expected capacity and network reliability can be readily determined by the following theorem.

Theorem 3. The expected capacity can be calculated as $\mathrm{E}$ $\mathrm{C}=\sum_{d=1}^{D}\left(R_{d}-R_{d+1}\right) \times d$.

Proof 3.

$$
\begin{aligned}
\operatorname{Pr}\{x \mid M(x) & =d\} \\
& =\operatorname{Pr}\{x \mid M(x) \geq d\}-\operatorname{Pr}\{x \mid M(x)>d\} \\
& =\operatorname{Pr}\{x \mid M(x) \geq d\}-\operatorname{Pr}\{x \mid M(x) \geq d+1\} \\
& =R_{d}-R_{d+1} .
\end{aligned}
$$

Thus,

$$
\mathrm{EC}=\sum_{d=1}^{D} \operatorname{Pr}\{x \mid M(x)=d\} \times d=\sum_{d=1}^{D}\left(R_{d}-R_{d+1}\right) \times d .
$$

In addition, it is noted that when $d=D, R_{d+1}=R_{D+1}=0$. Theorem 3 indicates that calculating EC is equivalent to the computation of $R_{d}$ for all possible $d$ values. Hence, the proposed $d$-minimal path algorithm is required to run multiple times for obtaining EC.

\section{A Case Study of a Coal Delivery Network}

Network reliability and expected capacity are two performance indicators for measuring the level of service of a freight network in a complex environment. In this section, we take a practical coal delivery network shown in Figure 3 as a case study to demonstrate the implications of both network reliability and expected capacity.

6.1. Performance Assessment in terms of Network Reliability. It is well known that coal is the largest source of energy in China and is used to generate a significant chunk of the nation's electricity. Every year, thousands of million tons of coal is transported from the producing area to the 
TABLE 2: Computational times of both algorithms.

\begin{tabular}{lccccccccc}
\hline Demand level $d$ & 9 & 10 & 11 & 12 & 13 & 14 & 15 & 16 & Average \\
\hline$T_{\text {new }}$ & 18.893 & 19.728 & 19.878 & 18.597 & 15.396 & 10.996 & 6.716 & 3.622 & 14.228 \\
$T_{\text {CL }}$ & 18.964 & 19.802 & 20.445 & 19.173 & 20.442 & 19.714 & 18.781 & 18.216 & 19.442 \\
\hline
\end{tabular}

Note: $T_{\mathrm{CL}}$ and $T_{\text {new }}$ are the computational times (in CPU seconds) consumed by Chen and Lin's algorithm [22] and the proposed algorithm, respectively.

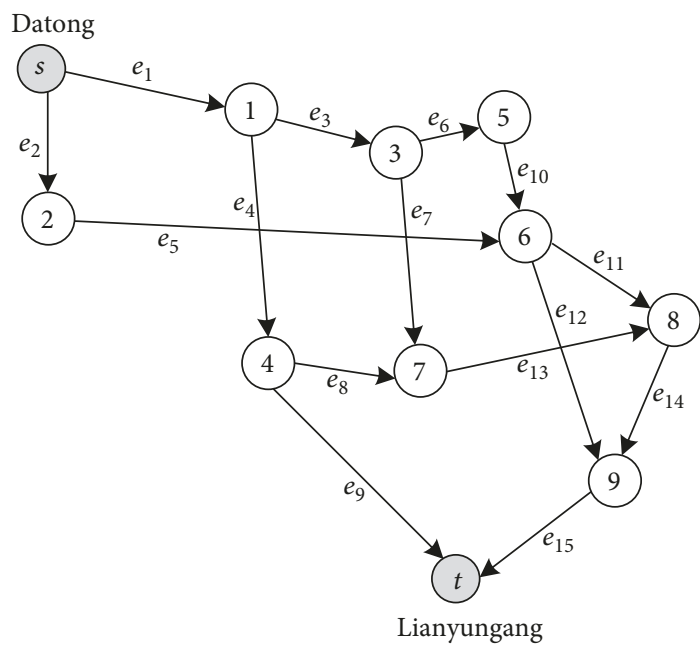

Figure 3: The coal delivery network from Datong to Lianyungang.

consumption area, so the efficient and safe movement of coal over space plays an extremely important part in sustaining the national economic development. Datong City in Shanxi Province that produces about 400 million tons of coal every year is a famous coal-producing area in China, and a large proportion of the produced coal is transported and sold all over the country. A producer owning a coal enterprise located at Datong City produces thermal coal. Thermal coal is usually used in power industry to generate electricity. Owing to price advantage, the producer has been one of the chief thermal coal suppliers for many Chinese power enterprises. One of its customers is a large power enterprise located at Lianyungang City in Jiangsu Province that is one of the richest provinces in China. Figure 3 describes the coal delivery network from Datong to Lianyungang, in which the coal commodity can pass through several transfer centers in different cities.

Coal is transported by train or ship. The capacity data on routes derived from the database of logistics service providers are provided in Table 3 . That is, each route has multiple possible carrying capacities, such as $0,1, \ldots, 5$ (unit: $10^{4}$ tons), following a given probability distribution. Supposing that the amount of coal ordered by the power enterprise at Lianyungang is $6 \times 10^{4}$ tons, i.e., demand level $d=6$ units of coal. The proposed algorithm is utilized to evaluate the network reliability $R_{6}$.

First, a total number of 41 6-minimal paths are obtained by using the proposed algorithm. Then, based on all 6-minimal paths and (1), the value of the performance index $R_{6}$ is calculated as 0.820714 . Therefore, the probability that the freight network in Figure 3 can successfully
TABle 3: Capacity data of each arc (route) in Figure 3.

\begin{tabular}{lcccccc}
\hline & \multicolumn{7}{c}{ Available capacity (unit: $10^{4}$ tons) } \\
Route $e_{i}$ & 0 & 1 & 2 & 3 & 4 & 5 \\
& & \multicolumn{5}{c}{ Probability } \\
\hline$e_{1}$ & 0.005 & 0.012 & 0.017 & 0.021 & 0.032 & 0.913 \\
$e_{2}$ & 0.011 & 0.043 & 0.946 & - & - & - \\
$e_{3}$ & 0.005 & 0.011 & 0.023 & 0.039 & 0.922 & \\
$e_{4}$ & 0.012 & 0.029 & 0.959 & - & - & \\
$e_{5}$ & 0.011 & 0.018 & 0.025 & 0.946 & - & \\
$e_{6}$ & 0.012 & 0.026 & 0.034 & 0.928 & - & \\
$e_{7}$ & 0.007 & 0.019 & 0.974 & - & - & \\
$e_{8}$ & 0.006 & 0.015 & 0.979 & - & - & \\
$e_{9}$ & 0.011 & 0.043 & 0.946 & - & - & \\
$e_{10}$ & 0.008 & 0.013 & 0.026 & 0.953 & - & \\
$e_{11}$ & 0.012 & 0.046 & 0.942 & - & - & \\
$e_{12}$ & 0.006 & 0.019 & 0.034 & 0.941 & - & \\
$e_{13}$ & 0.007 & 0.012 & 0.029 & 0.952 & - & - \\
$e_{14}$ & 0.009 & 0.016 & 0.029 & 0.035 & 0.911 & \\
$e_{15}$ & 0.006 & 0.011 & 0.015 & 0.022 & 0.034 & 0.912 \\
\hline
\end{tabular}

deliver $6 \times 10^{4}$ tons of coal commodities from Datong to Lianyungang is 0.820714 . Undoubtedly, the reliability value 0.820714 reveals valuable knowledge on the operational performance of the delivery network and can be regarded as a decision criterion. For example, if the value 0.820714 is below the expectation of the manager, it means that the service performance of the network does not meet the requirement; otherwise, the service performance of the network is acceptable.

6.2. Performance Assessment in terms of Expected Capacity. By Equation (22), it needs to compute network reliabilities at different demand levels in order to obtain expected capacity EC. Obviously, the largest network capacity is $D=7$. Thus, the proposed algorithm is utilized to compute network reliabilities at different demand levels from 1 to 7 . The results are summarized in Table 4. Moreover, the reliability difference between two neighboring demand levels is calculated and adopted to compute EC. The final computational results are also presented in Table 4 by which we make the following observations:

(1) As expected, network reliability declines as the demand level $d$ rises. In addition, the reliability difference between two neighboring demand levels $\left(R_{d}-R_{d+1}\right)$ increases as the demand level $d$ increases 
TABLE 4: Network reliabilities at different demand levels and expected capacity.

\begin{tabular}{lccccccc}
\hline Demand level $d$ & 1 & 2 & 3 & 4 & 5 & 6 & 7 \\
\hline$R_{d}$ & 0.999721 & 0.998355 & 0.985891 & 0.960840 & 0.919381 & 0.820714 & 0.628040 \\
$R_{d}-R_{d+1}$ & 0.001366 & 0.012464 & 0.025051 & 0.041459 & 0.098667 & 0.192674 & 0.628040 \\
$\left(R_{d}-R_{d+1}\right) \times d$ & 0.001366 & 0.024928 & 0.075153 & 0.165836 & 0.493335 & 1.156044 & 4.39628 \\
Expected capacity & & & & $\mathrm{EC}=6.312942$ & & \\
\hline
\end{tabular}

(2) For the demand level $d=6$, it is noteworthy that the expected capacity $\mathrm{EC}=6.312942$ is larger than demand level $d=6$, which means the service performance of the network is relatively desirable from the perspective of the average network capacity level

In summary, both network reliability and expected capacity can be adopted to assess the performance level of a freight network from different aspects, which provides managers useful tools for making reasonable evaluation on the operational state of their freight network.

\section{Conclusions}

Reliability evaluation assists supervisors in understanding network service performance. This paper is devoted to performance assessment of a freight network in the light of reliability analysis. On one hand, this paper presents an efficient $d$-minimal path-based algorithm to evaluate the freight network reliability that at least $d$ units of commodity demand can be successfully delivered from the source to the destination. An improved model is constructed by redefining capacity bounds of arcs and minimal paths to enhance the efficiency of solving $d$-minimal paths. On the other hand, this paper puts forward a new concept of expected capacity to assess the service performance of a freight network. In addition, a practical case study regarding a coal delivery network is provided to demonstrate the implications of both network reliability and expected capacity.

For future research, there is still potential for developing a new reliability model to meet practical needs. For example, we only consider the transmission of a single type of commodity in the freight network, and yet, it is much more valuable to assess the performance of multicommodity freight networks in which multiple types of cargoes are delivered from the source to the destination simultaneously.

\section{Data Availability}

This article is supported by the data which are openly available at locations cited in the reference section.

\section{Conflicts of Interest}

The authors declare that they have no conflicts of interest.

\section{Acknowledgments}

This work is mainly supported by the National Natural Science Foundation of China (Project Nos. 71601072 and 61872126), the Research Project from the Science and Technology Department of Henan Province (Project No. 172102310677), the Fundamental Research Funds for the Universities of Henan Province (Project No. NSFRF170914), and the Doctoral Research Funds of Henan Polytechnic University (Project No. B2018-51).

\section{References}

[1] A. Costa, G. Celano, S. Fichera, and E. Trovato, "A new efficient encoding/decoding procedure for the design of a supply chain network with genetic algorithms," Computers \& Industrial Engineering, vol. 59, no. 4, pp. 986-999, 2010.

[2] M. Yu and A. Nagurney, "Competitive food supply chain networks with application to fresh produce," European Journal of Operational Research, vol. 224, no. 2, pp. 273-282, 2013.

[3] S. Zhang, X. Li, and C. Zhang, "A fuzzy control model for restraint of bullwhip effect in uncertain closed-loop supply chain with hybrid recycling channels," IEEE Transactions on Fuzzy Systems, vol. 25, no. 2, pp. 475-482, 2017.

[4] S. Zhang, Y. Hou, S. Zhang, and M. Zhang, "Fuzzy control model and simulation for nonlinear supply chain system with lead times," Complexity, vol. 2017, Article ID 2017634, 11 pages, 2017.

[5] A. Nagurney, S. Saberi, S. Shukla, and J. Floden, "Supply chain network competition in price and quality with multiple manufacturers and freight service providers," Transportation Research Part E: Logistics and Transportation Review, vol. 77, pp. 248-267, 2015.

[6] M. G. Cedillo-Campos, J. L. de la Riva-Canizales, A. BuenoSolano, J. Gonzalez-Feliu, and J. L. García-Alcaraz, "Reliability in urban freight distribution: a Markovian approach," DYNA, vol. 81, no. 187, pp. 232-239, 2014.

[7] Y. K. Lin, C. T. Yeh, and C. F. Huang, "Reliability assessment of a multistate freight network for perishable merchandise with multiple suppliers and buyers," International Journal of Systems Science, vol. 48, no. 1, pp. 74-83, 2016.

[8] Y.-K. Lin, "System reliability evaluation for a multistate supply chain network with failure nodes using minimal paths," IEEE Transactions on Reliability, vol. 58, no. 1, pp. 34-40, 2009.

[9] C. T. Yeh, Y. K. Lin, and C. F. Huang, "A reliability indicator to measure a stochastic supply chain network with transportation damage and limited production capacity," IIE Transactions, vol. 46, no. 10, pp. 1066-1078, 2014.

[10] C. C. Jane, "Performance evaluation of logistics systems under cost and reliability considerations," Transportation Research 
Part E: Logistics and Transportation Review, vol. 47, no. 2, pp. 130-137, 2011.

[11] C. C. Jane and Y. W. Laih, "Evaluating cost and reliability integrated performance of stochastic logistics systems," Naval Research Logistics, vol. 59, no. 7, pp. 577-586, 2012.

[12] Y. F. Niu, Z. Y. Gao, and W. H. K. Lam, "Evaluating the reliability of a stochastic distribution network in terms of minimal cuts," Transportation Research Part E: Logistics and Transportation Review, vol. 100, pp. 75-97, 2017.

[13] Y.-F. Niu, W. H. K. Lam, and Z. Gao, "An efficient algorithm for evaluating logistics network reliability subject to distribution cost," Transportation Research Part E: Logistics and Transportation Review, vol. 67, pp. 175-189, 2014.

[14] J. S. Lin, C. C. Jane, and J. Yuan, "On reliability evaluation of a capacitated-flow network in terms of minimal pathsets," Network, vol. 25, no. 3, pp. 131-138, 1995.

[15] Y. K. Lin, "A simple algorithm for reliability evaluation of a stochastic-flow network with node failure," Computers \& Operations Research, vol. 28, no. 13, pp. 1277-1285, 2001.

[16] W. C. Yeh, "A simple method to verify all d-minimal path candidates of a limited-flow network and its reliability," The International Journal of Advanced Manufacturing Technology, vol. 20, no. 1, pp. 77-81, 2002.

[17] W. C. Yeh, "A novel method for the network reliability in terms of capacitated minimum-paths without knowing minimum-paths in advance," Journal of the Operational Research Society, vol. 56, no. 10, pp. 1235-1240, 2017.

[18] W. C. Yeh, "Evaluating the reliability of a novel deteriorationeffect multi-state flow network," Information Sciences, vol. 243, pp. 75-85, 2013.

[19] Y. F. Niu, Z. Y. Gao, and W. H. K. Lam, "A new efficient algorithm for finding all $d$-minimal cuts in multi-state networks," Reliability Engineering and System Safety, vol. 166, pp. 151-163, 2017.

[20] Y. F. Niu and X. Z. Xu, "Reliability evaluation of multi-state systems under cost consideration," Applied Mathematical Modelling, vol. 36, no. 9, pp. 4261-4270, 2012.

[21] Y. Niu, Z. Gao, and H. Sun, "An improved algorithm for solving all $d$-MPs in multi-state networks," Journal of Systems Science and Systems Engineering, vol. 26, no. 6, pp. 711-731, 2017.

[22] S. G. Chen and Y. K. Lin, "Searching for $d$-MPs with fast enumeration," Journal of Computational Science, vol. 17, pp. 139-147, 2016.

[23] L. R. Ford and D. R. Fulkerson, Flows in Networks, Princeton University Press, New Jersey, 1962.

[24] R. K. Ahuja, M. Kodialam, A. K. Mishra, and J. B. Orlin, "Computational investigations of maximum flow algorithms," European Journal of Operational Research, vol. 97, no. 3, pp. 509-542, 1997.

[25] S.-G. Chen and Y.-K. Lin, "Search for all minimal paths in a general large flow network," IEEE Transactions on Reliability, vol. 61, no. 4, pp. 949-956, 2012.

[26] G. Bai, Z. Tian, and M. J. Zuo, "An improved algorithm for finding all minimal paths in a network," Reliability Engineering \& System Safety, vol. 150, pp. 1-10, 2016.

[27] W.-C. Yeh, "A simple universal generating function method to search for all minimal paths in networks," IEEE Transactions on Systems, Man, and Cybernetics - Part A: Systems and Humans, vol. 39, no. 6, pp. 1247-1254, 2009.
[28] S. G. Chen, "Efficiency improvement in explicit enumeration for integer programming problems," in 2013 IEEE International Conference on Industrial Engineering and Engineering Management, pp. 98-100, Bangkok, Thailand, December 2013.

[29] D. Shier, Network Reliability and Algebraic Structures, Clarendon Press, New York, 1991. 


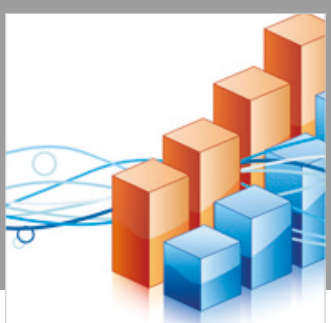

Advances in

Operations Research

\section{-n-m}
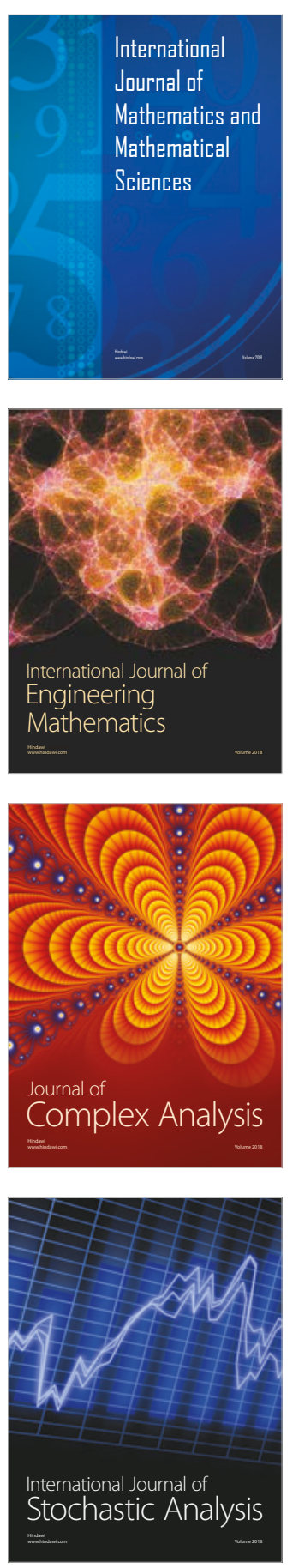
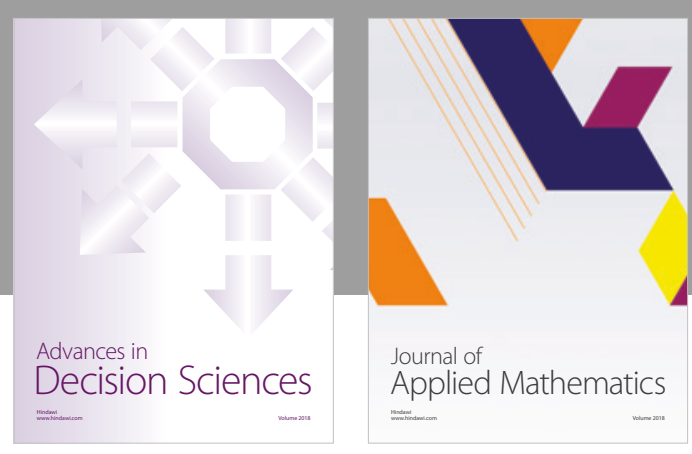

Journal of

Applied Mathematics
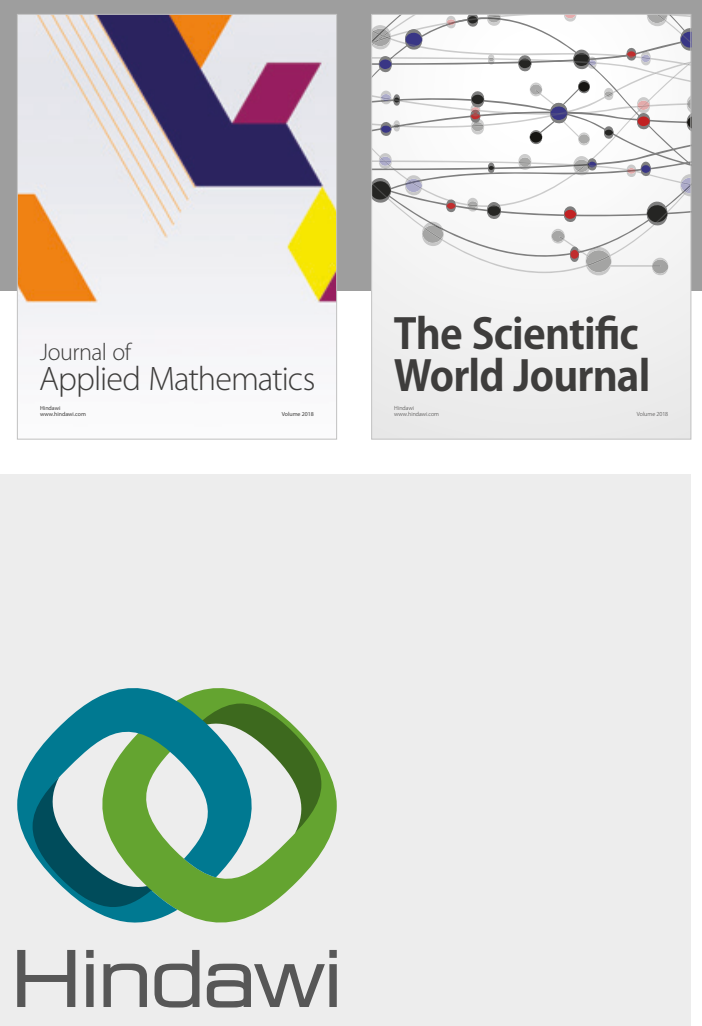

Submit your manuscripts at

www.hindawi.com

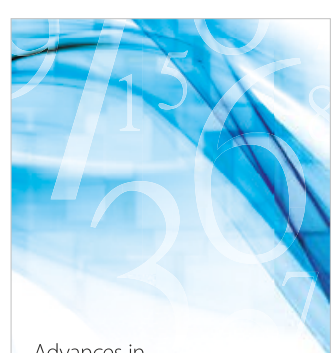

Advances in
Numerical Analysis
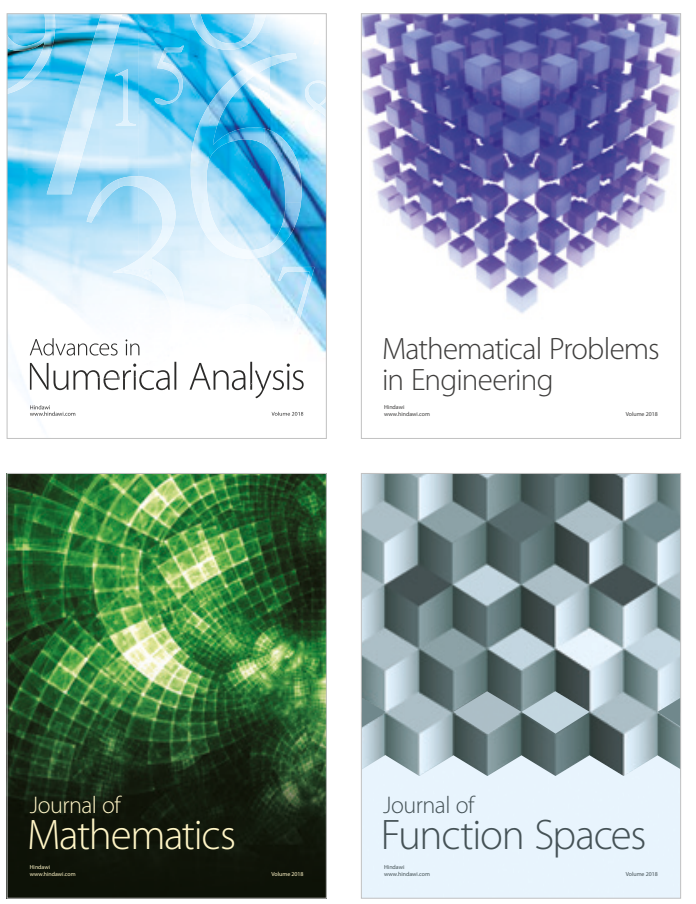

Mathematical Problems in Engineering

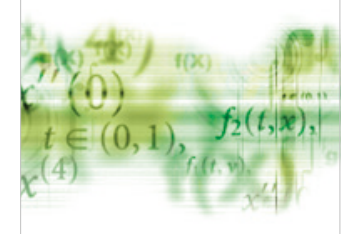

International Journal of

Differential Equations

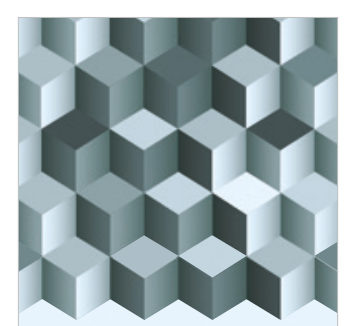

Journal of

Function Spaces
The Scientific

World Journal

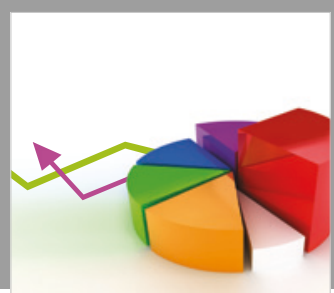

Journal of

Probability and Statistics
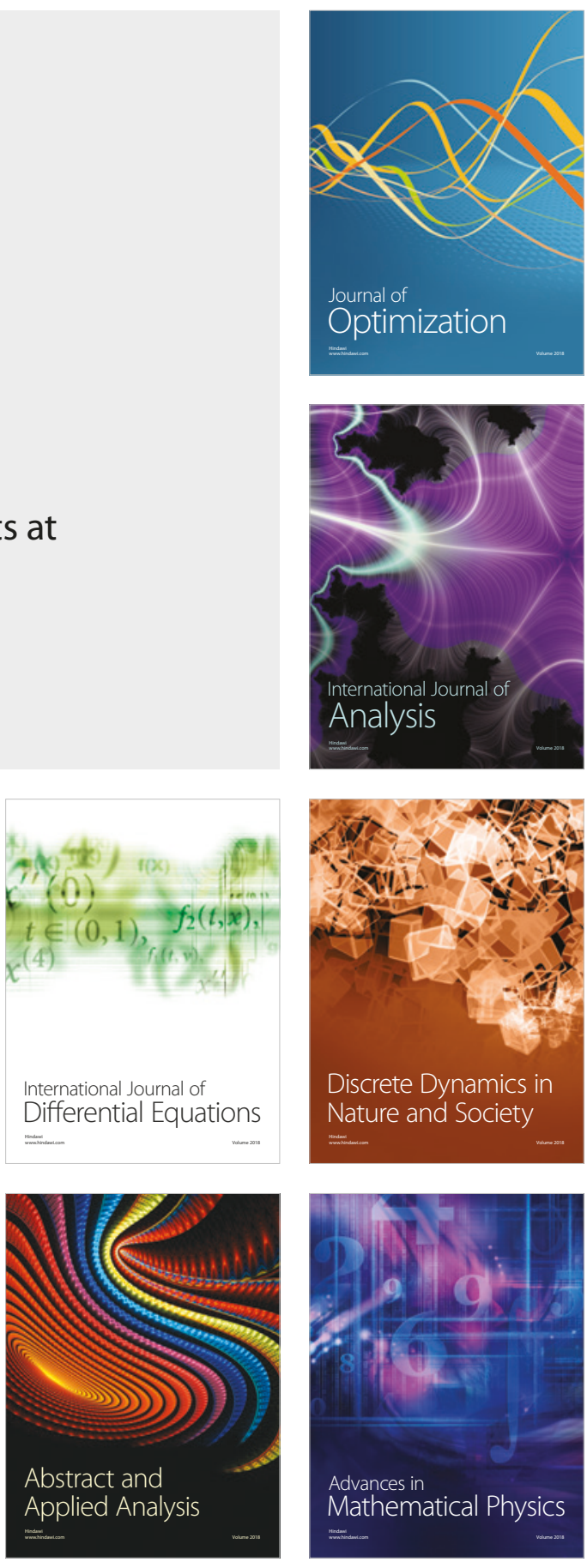